OPEN ACCESS

Edited by:

Fabio Grizzi,

Humanitas Research Hospital, Italy

Reviewed by:

Nida Zahid,

Aga Khan University, Pakistan

Patrizia Mondello,

Memorial Sloan Kettering Cancer

Center, United States

*Correspondence:

Qiang Dong

dqiang666@163.com

Specialty section:

This article was submitted to

Genitourinary Oncology,

a section of the journal

Frontiers in Oncology

Received: 24 September 2020 Accepted: 04 May 2021

Published: 14 July 2021

Citation:

Liu Z-H, Yang L-C, Song P, Fang K, Zhou J, Peng Z-F and Dong Q (2021)

Primary Diffuse Large B-Cell

Lymphoma of the Urinary Tract: A

Population-Based Analysis.

Front. Oncol. 11:609882.

doi: 10.3389/fonc.2021.609882

\section{Primary Diffuse Large B-Cell Lymphoma of the Urinary Tract: A Population-Based Analysis}

\author{
Zheng-Huan Liu, Lu-Chen Yang, Pan Song, Kun Fang, Jing Zhou, Zhu-Feng Peng \\ and Qiang Dong * \\ Department of Urology, Institute of Urology, West China Hospital, Sichuan University, Chengdu, China
}

Objective: Diffuse large B-cell lymphoma (DLBCL) is the most common histopathological type of non-Hodgkin's lymphoma, which may arise from various extranodal sites. Little is known about the clinical characteristics and survival outcomes of primary DLBCL of the urinary tract (UT). Thus, we conducted this study to explore the independent prognostic factors of patients with UT-DLBCL using the Surveillance, Epidemiology, and End Results (SEER) database.

Materials and Methods: We searched the Surveillance, Epidemiology, and End Results (SEER) database for the data of patients diagnosed with UT-DLBCL between 1975 and 2016. Data, including demographic tumour stage and therapeutic strategies, such as surgical resection, radiation therapy, and chemotherapy, were collected. The impact of these factors on survival outcomes, including overall survival (OS) and disease-specific survival (DSS), was analysed using Kaplan-Meier curves.

Results: Four-hundred and eighty-nine patients who met the inclusion criteria were enrolled in the data analysis. The median age was 69 years old. Most cases of UT-DLBCL (72.39\%) originated from the kidney, followed by the urinary bladder (24.95\%). Both surgical resection and chemotherapy can significantly improve OS and DSS. Patients older than 75 years had the worst survival outcomes. Stage IV DLBCL may be a poor prognostic factor.

Conclusion: To the best of our knowledge, this is the largest population-based study of UT-DLBCL. Advanced age, male gender, lack of surgical resection or chemotherapy, and stage IV DLBCL were poor prognostic factors.

Keywords: diffuse large B-cell lymphoma, urinary tract, SEER, prognosis, primary

\section{INTRODUCTION}

The most commonly diagnosed non-Hodgkin's lymphoma (NHL) subtype is diffuse large B-cell lymphoma (DLBCL), which accounts for approximately $30 \%$ of NHL cases (1). Lymphomas can arise in almost every organ or site, and approximately one-third of the patients have extranodal origins (2). Among them, less than 5\% were genito-urinary lymphomas (3). Furthermore, lymphomas of the UT are extremely rare; no more than 100 cases have been reported so far 
globally (4). The etiological exposure, clinical characteristics, and survival outcomes may vary for different sites of origin (5).

The use of recently established first-line therapy, including rituximab combined with cyclophosphamide, doxorubicin, vincristine, and prednisone (R-CHOP), has improved the survival outcomes of patients with DLBCL (6). However, questions about the treatment-related side effects, as well as long-term complications, remain unanswered. Increasing evidence has shown that extranodal sites demonstrate distinct clinical characteristics, survival outcomes, and require specific treatment $(7,8)$. Thus, studies on the clinical features and survival outcomes of DLBCL originating from the UT are limited.

This study explored the prognostic factors of patients with UT-DLBCL, based on data from the SEER database, to provide findings that will facilitate better clinic evaluations and more effective management of DLBCL.

\section{MATERIALS AND METHODS}

We utilized the SEER database to enrol eligible patients between 1975 and 2016, collected from 18 state registries in the USA. The patients diagnosed with lymphoma with the third edition of the International Classification of Diseases for Oncology (ICD-O-3) histology code of 9680 (DLBCL, not otherwise specified) in the database were included. Patients with DLBCL originating from sites other than the UT were excluded. In addition, all cases diagnosed before 1983 were excluded for the lack of the Ann-Arbor stage information. The patients without active follow-up and unknown Ann-Arbor stage, and those without histopathological confirmation of DLBCL, were also excluded from this study. Eligible patients were further identified based on the primary site, such as the kidney, ureter, urinary bladder, and urethra.

All the demographic, clinical, pathological, and survival data of the patients were extracted from the SEER database. The patients were divided into two groups based on the primary site: upper UT (UUT) group, with sites that include kidney, renal pelvis, and ureter; and lower UT (LUT) group, with sites that include urinary bladder and urethra. The treatments for patients with UT-DLBCL were also exported from the database, including radiotherapy, cancer-directed surgical resection, and chemotherapy. The ages of diagnosis were grouped into the following: $0-60,60-75$, and older than 75 years. The races were classified into white, black, and other. Importantly, the overall survival (OS) and disease-specific survival (DSS) were both analysed in this study.

The SEER database records survival as the number of months elapsed from the time of diagnosis of DLBCL to the day of death or the latest contact. For the estimation of disease-specific survival (DSS), only death attributable to the DLBCL was considered an exact event. To prevent confounding, we excluded patients diagnosed with more than one primary malignancy from the survival analysis.

\section{Ethical Approval}

All data used in this study were from the public database, thus, ethical approval was required. We maintained the confidentiality of the data and reported the study results according to the SEER Research Data Use Agreement.

\section{Statistical Analysis}

Continuous variables with normal distribution are presented as mean and standard deviation (SD); otherwise, median and interquartile range are considered. The differences in continuous variables were evaluated using the 2-sided analysis of variance (ANOVA) or the Mann-Whitney U-test according to their distribution. The categorical variables are presented as counts and percentages. Chi-squares or Fischer's exact tests were applied to evaluate their differences. The survival curves were estimated using the Kaplan-Meier method and compared using the log-rank test. Cox proportional hazard models were used to identify the independent predictors of disease-specific mortality. Patients with missing data for one or more of the variables were excluded from the multivariable regression analysis. To allow adequate power for the Kaplan-Meier curves and the Cox model, the kidney cases were grouped with renal pelvis and ureter cases, whereas the urethral cases were grouped with the bladder cases. The statistical analysis was performed using IBM SPSS Statistics 24 software (IBM Corp., Armonk, NY, USA), and a p-value of less than 0.05 was considered statistically significant.

\section{RESULTS}

\section{Demographics and Clinical Features}

Finally, we enrolled 489 eligible patients diagnosed with UTDLBCL between 1975 and 2016 from the SEER database. The demographic and clinical characteristics of the patients are summarized in Table 1. The mean age at diagnosis was 69 years. There were 143 (29.24), 166 (33.95\%), and 180 (36.81\%) patients in the $<60,60-75$, and $\geq 75$ years age-at-diagnosis groups, respectively. The gender distributions were not different. Most of the patients were white $(84.04 \%) ; 15.54 \%$ were black or other. The most common primary site was the kidney $(72.39 \%)$, followed by the urinary bladder (UB) (24.95\%).

Surprisingly, cancer-directed surgery was performed in about half of the cases (56.44\%). Forty-one patients (7.77\%) underwent radiation therapy. The majority of these cases $(72.80 \%)$ underwent chemotherapy. The most common Ann-Arbor stage at presentation was stage I $(34.15 \%)$, followed by stage IV (34.35\%).

\section{Prognostic Factors of Survival Outcomes}

Patients aged $\geq 75$ years had the worst survival outcomes, with OS of only 8 months and DSS of 12 months (Figure 1 and Table 2, $\mathrm{p}<0.001$ ). The 5 -year OS for the $<60$ and $60-75$ years age-at-diagnosis groups of patients were $64.29 \%$ and $43.51 \%$, respectively; the 5 -year OS was $27.10 \%$ for the $\geq 75$ years group. The 5-year DSS for these three groups were $69.06 \%, 51.36 \%$, and $39.23 \%$, respectively.

Additionally, we found no differences in OS or DSS in the gender and race groups (Table 2). There was also no difference between the survival outcomes of the UUT and LUT groups 
TABLE 1 | Characteristics of patients with DLBCL ( $\mathrm{N}=489)$.

\begin{tabular}{|c|c|}
\hline & Number of cases (\%) \\
\hline Age at diagnosis (range) & $69(4-97)$ \\
\hline$<60$ & $143(29.24)$ \\
\hline $60-75$ & 166 (33.95) \\
\hline$\geq 75$ & $180(36.81)$ \\
\hline \multicolumn{2}{|l|}{ Gender } \\
\hline Female & 221 (45.19) \\
\hline Male & $268(54.81)$ \\
\hline \multicolumn{2}{|l|}{ Marriage status } \\
\hline Married & $259(52.97)$ \\
\hline Single & $211(43.15)$ \\
\hline Unknown & 19 (3.88) \\
\hline \multicolumn{2}{|l|}{ Race } \\
\hline White & $411(84.04)$ \\
\hline Black & $38(7.77)$ \\
\hline Other & $38(7.77)$ \\
\hline Unknown & $2(0.42)$ \\
\hline \multicolumn{2}{|l|}{ Primary site } \\
\hline Kideny & 354 (72.39) \\
\hline Renal Pelvis & $3(0.61)$ \\
\hline Ureter & $4(0.82)$ \\
\hline Urinary Bladder & $122(24.95)$ \\
\hline Urethral & $6(1.23)$ \\
\hline \multicolumn{2}{|l|}{ Surgery } \\
\hline Yes & $213(43.56)$ \\
\hline No & $276(56.44)$ \\
\hline \multicolumn{2}{|l|}{ Radiation } \\
\hline Yes & $38(7.77)$ \\
\hline No & 451 (92.23) \\
\hline \multicolumn{2}{|l|}{ Chemotherapy } \\
\hline Yes & $356(72.80)$ \\
\hline No & $133(27.20)$ \\
\hline \multicolumn{2}{|l|}{ Ann Arbor Stage } \\
\hline Stage I & $167(34.15)$ \\
\hline Stage ॥ & $119(24.34)$ \\
\hline Stage III & $35(7.16)$ \\
\hline Stage IV & $168(34.35)$ \\
\hline
\end{tabular}

DLBCL, Diffuse large B cell lymphoma.

(Table 2). Regarding the treatment strategies, patients who had undergone cancer-directed surgery had significantly longer OS than those who had not (Figure 2A and Table 2, 63 vs. 16 months, HR: 1.310, 95\%CI: 1.051-1.633, p=0.016). The same outcome was found for DSS in the two groups (Figure $\mathbf{2 B}$ and Table 2, HR: 1.624, 95\%CI: 1.241-2.127, p < 0.001), whereas radiotherapy had no survival benefit to patients with UTDLBCL. Not surprisingly, patients who were not undergoing chemotherapy had approximately twice the risk of death as those who were (Figure 3 and Table 2, p < 0.001). Finally, we found that patients with Stage IV DLBCL had worse survival KaplanMeier curves than the others (Figure 4 and Table 2).

\section{Multivariate Survival Analysis}

The outcomes of the multivariate survival analysis are summarized in Table 3. Variables, including age, gender, primary site, treatment strategies, and Ann-Arbor stage, were analysed. Older patients had worse OS and DSS than younger patients. Different from the above results, males had worse OS (HR: 1.336, 95\% CI: 1.061-1.681, p=0.014) and DSS (HR: 1.340, 95\%CI: $1.020-1.759, \mathrm{p}=0.036)$. Patients who had undergone surgical resection had better OS and DSS than those who had not $(\mathrm{p}<0.05)$. In addition, chemotherapy had a remarkable effect on survival in UT-DLBCL $(\mathrm{p}<0.001)$. Patients with stage IV UTDLBCL tended to have worse survival than those with Stage I UT-DLBCL; this was not only for OS (HR:1.606, 95\%CI: 1.2022.146, $\mathrm{p}=0.001$ ) but also for DSS (HR:1.835, 95\%CI: 1.298-2.594, $\mathrm{p}=0.001)$. The other variables did not affect the survival outcomes of UT-DLBCL.

\section{Subgroup Analysis of Kidney Versus UB}

Patients with kidney DLBCL were younger than those with UBDLBCL (Table 4, p < 0.001). Kidney DLBCL was predominant in males (59.89\%), and UB-DLBCL was predominant in females (59.84\%). More than half of the cases of UB-DLBCL $(56.56 \%)$ were diagnosed as Stage I, compared with only $25.71 \%$ of the cases of kidney DLBCL ( $\mathrm{p}<0.001$ ). Interestingly, for patients with DLBCL of the UB, cancer-directed surgery had no survival benefit (Figure 5, $\mathrm{p}=0.593$ ), which was different from those with kidney DLBCL (Figure 6, $\mathrm{p}=0.005$ ).

\section{DISCUSSION}

Lymphomas can arise from any site, and extranodal lymphomas account for one-third of all cases (2). DLBCL is the most common subtype of NHL. However, lymphomas arising from the UT account for less than $5 \%$ of the extranodal NHL (3). For instance, less than 100 cases of UT lymphomas have been reported so far (4). Thus, UT-DLBCL is an extremely rare
A

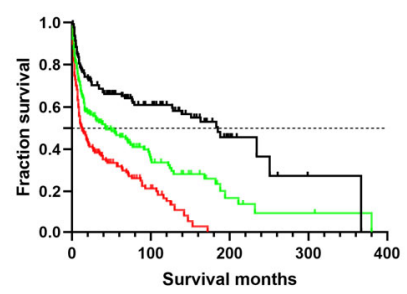

B

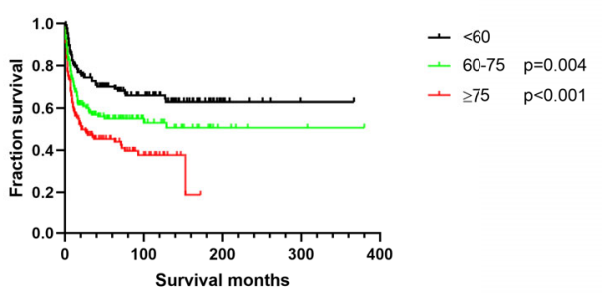

FIGURE 1 | Survival analysis based on age: (A) overall survival; (B) disease-specific survival. 
TABLE 2 | Univariate survival analysis.

\begin{tabular}{|c|c|c|c|c|c|c|}
\hline & os & HR (95\% Cl) & $p$-value & DSS & HR $(95 \%$ Cl) & p-value \\
\hline \multicolumn{7}{|l|}{ Age } \\
\hline$<60$ & 185 & Reference & & Not reached & Reference & \\
\hline $60-75$ & 31 & $2.080(1.529-2.830)$ & $<0.001^{\star}$ & 100 & $1.703(1.187-2.443)$ & $0.004^{*}$ \\
\hline$\geq 75$ & 8 & 3.564 (2.634-4.822) & $<0.001^{\star}$ & 12 & 2.671 (1.893-3.770) & $<0.001^{*}$ \\
\hline \multicolumn{7}{|l|}{ Gender } \\
\hline Female & 43 & Reference & & Not reached & Reference & \\
\hline Male & 17 & $1.226(0.985-1.527)$ & 0.069 & 35 & $1.282(0.986-1.666)$ & 0.063 \\
\hline \multicolumn{7}{|c|}{ Marital status } \\
\hline Married & 30 & Reference & & 71 & Reference & \\
\hline Single & 22 & $1.068(0.854-1.335)$ & 0.566 & 100 & $1.011(0.776-1.316)$ & 0.937 \\
\hline \multicolumn{7}{|l|}{ Race } \\
\hline White & 27 & Reference & & 76 & Reference & \\
\hline Black & 25 & $0.926(0.614-1.397)$ & 0.715 & 100 & 0.999 (0.616-1.620) & 0.998 \\
\hline Other & 17 & $1.128(0.754-1.687)$ & 0.558 & 72 & $1.188(0.750-1.883)$ & 0.463 \\
\hline \multicolumn{7}{|l|}{ Primary site } \\
\hline UUT & 26 & Reference & & 67 & Reference & \\
\hline LUT & 27 & $1.080(0.848-1.376)$ & 0.533 & 128 & $0.892(0.660-1.205)$ & 0.455 \\
\hline \multicolumn{7}{|l|}{ Surgery } \\
\hline Yes & 63 & Reference & & Not reached & Reference & \\
\hline No & 16 & $1.310(1.051-1.633)$ & $0.016^{\star}$ & 21 & $1.624(1.241-2.127)$ & $<0.001^{*}$ \\
\hline \multicolumn{7}{|l|}{ Radiation } \\
\hline Yes & 87 & Reference & & Not reached & Reference & \\
\hline No & 21 & $1.419(0.936-2.152)$ & 0.099 & 63 & 1.995 (1.116-3.568) & $0.020^{*}$ \\
\hline \multicolumn{7}{|c|}{ Chemotherapy } \\
\hline Yes & 57 & Reference & & 153 & Reference & \\
\hline No & 3 & $1.998(1.583-2.522)$ & $<0.001^{*}$ & 4 & $2.241(1.708-2.940)$ & $<0.001^{\star}$ \\
\hline \multicolumn{7}{|c|}{ Ann Arbor Stage } \\
\hline Stage I & 57 & Reference & & Not reached & Reference & \\
\hline Stage ॥ & 77 & $0.927(0.684-1.257)$ & 0.625 & Not reached & $1.004(0.691-1.460)$ & 0.982 \\
\hline Stage III & 21 & $1.261(0.815-1.951)$ & 0.297 & 24 & 1.439 (0.873-2.370) & 0.153 \\
\hline Stage IV & 10 & $1.537(1.188-1.987)$ & $0.001^{\star}$ & 13 & $1.827(1.340-2.489)$ & $<0.001^{*}$ \\
\hline
\end{tabular}

OS, Overall survival; DSS, disease specific survival; HR, Hazard ratio; Cl, Confidence interval; UUT, Upper Urinary Tract; LUT, Lower Urinary Tract; ${ }^{\star} p$ < 0.05.
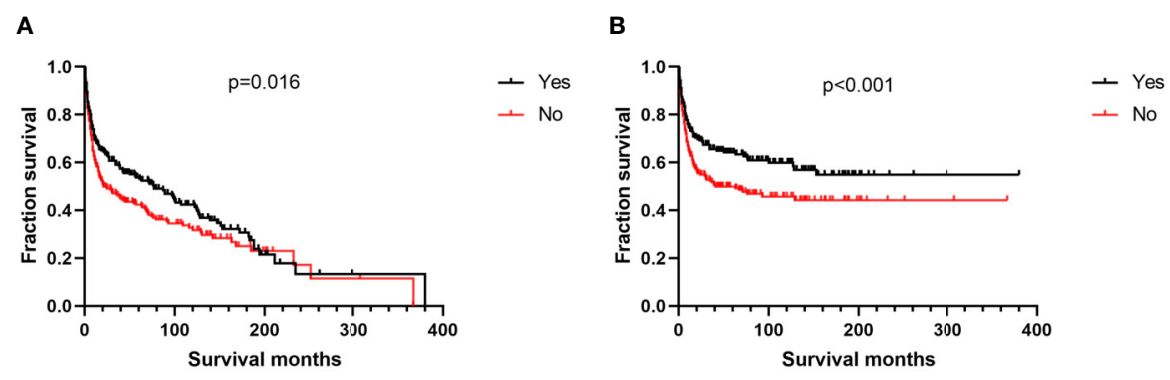

FIGURE 2 | Survival analysis based on surgical resection: (A) overall survival; (B) disease-specific survival.

NHL. As far as we know, this is the first study to explore the clinical features and survival outcomes for UT-DLBCL in a relatively large population, and this may help urologists manage UT-DLBCL better.

In this study, we found that patients aged $\geq 60$ years were more likely to suffer from UT-DLBCL. In previous studies, a multistep carcinogenic model of solid tumours (9) showed that the incidence of UT-DLBCL increases exponentially with aging. Two reasons may contribute to this phenomenon: first, the incidence of chronic inflammation can gradually increase with aging, which is one of the major risk factors of mortality (10).
Secondly, the immune function of older patients may decline. The deregulation of the immune system is characterised by thymic involution, which leads to a gradual decline in the development of naïve $\mathrm{T}$ cells (11). Moreover, several genes or molecules change with aging, and this may promote the development of DLBCL (12).

Importantly, we found that age was an independent prognostic factor of OS and DSS. The morality of patients aged $\geq 75$ years was $2-3$ times higher than that of younger patients. A previous study demonstrated that older age is a poor prognostic factor of UT lymphomas (13). 
A

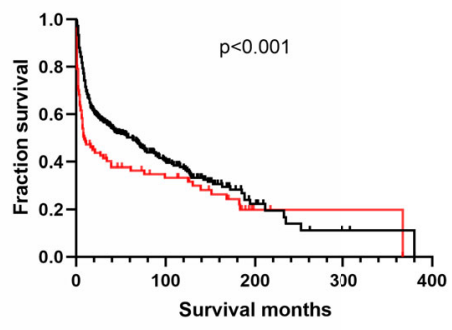

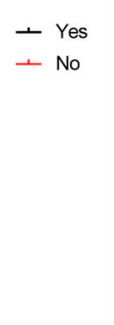

B

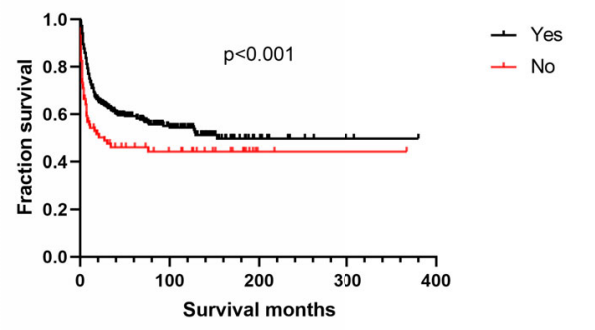

FIGURE 3 | Survival analysis based on chemotherapy: (A) overall survival; (B) disease-specific survival.

A

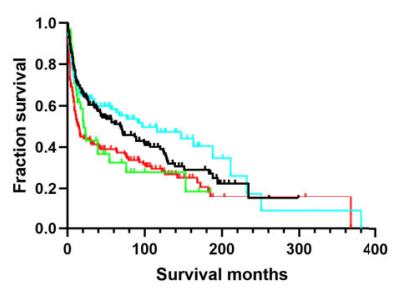

B

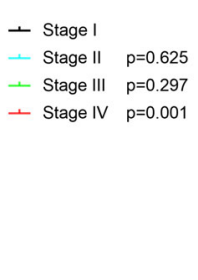

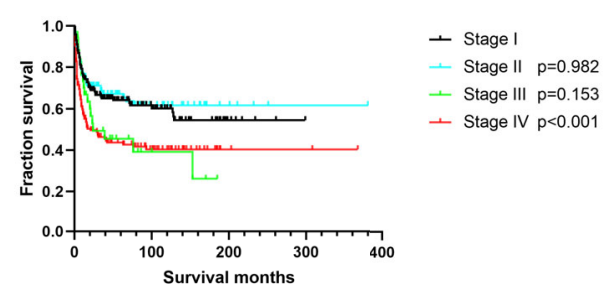

FIGURE 4 | Survival analysis based on Ann-Arbor stage: (A) overall survival; (B) disease-specific survival.

TABLE 3 | Multivariate survival analysis.

os

\section{HR $(95 \%$ CI)}

Reference

$2.253(1.646-3.086)$

$3.592(2.639-4.890)$

Reference

$1.336(1.061-1.681)$

Reference

$1.248(0.940-1.657)$

Reference

$1.301(1.014-1.670)$

Reference

$1.315(0.829-2.087)$

Reference

$2.031(1.595-2.585)$

Reference

1.013 (0.736-1.395)

$1.113(0.714-1.736)$

1.606 (1.202-2.146) p-value

$<0.001^{*}$

$<0.001^{*}$

$0.014^{*}$

0.125

$0.039^{*}$

0.245

$<0.001^{\star}$

Ann Arbor Stage

Stage I

Stage II

Stage III

Stage IV

0.636

$0.001^{*}$
DSS

\begin{tabular}{|c|c|}
\hline \multicolumn{2}{|c|}{ DSS } \\
\hline HR (95\% Cl) & $p$-value \\
\hline \multicolumn{2}{|l|}{ Reference } \\
\hline $1.815(1.260-2.615)$ & $0.001^{*}$ \\
\hline $2.645(1.863-3.755)$ & $<0.001^{*}$ \\
\hline \multicolumn{2}{|l|}{ Reference } \\
\hline $1.340(1.020-1.759)$ & $0.036^{\star}$ \\
\hline \multicolumn{2}{|l|}{ Reference } \\
\hline $1.183(0.837-1.671)$ & 0.341 \\
\hline \multicolumn{2}{|l|}{ Reference } \\
\hline $1.539(1.136-2.084)$ & $0.005^{*}$ \\
\hline \multicolumn{2}{|l|}{ Reference } \\
\hline $1.521(0.810-2.858)$ & 0.192 \\
\hline \multicolumn{2}{|l|}{ Reference } \\
\hline 2.405 (1.813-3.191) & $<0.001^{*}$ \\
\hline \multicolumn{2}{|l|}{ Reference } \\
\hline 1.069 (0.723-1.580) & 0.738 \\
\hline $1.325(0.795-2.206)$ & 0.280 \\
\hline $1.835(1.298-2.594)$ & $0.001^{*}$ \\
\hline
\end{tabular}

OS, Overall survival; DSS, disease specific survival; HR, Hazard ratio; Cl, Confidence interval; UUT, Upper Urinary Tract; LUT, Lower Urinary Tract; ${ }^{\circledR} \mathrm{P}<0.05$. 
TABLE 4 | Subgroup analysis of patients with kidney versus UB DLBCL.

\begin{tabular}{llccc}
\hline Characteristics & Kidney & UB & p-value \\
\hline Age, years & Median & 68 & 76 & $<0.001 \mathrm{a}^{*}$ \\
Gender, n & Female & 142 & 73 & $<0.001 \mathrm{~b}^{*}$ \\
\multirow{2}{*}{ Ann-Arbor stage, $\mathrm{n}$} & Male & 212 & 49 & \\
& I & 91 & 69 & $<0.001 \mathrm{~b}^{*}$ \\
& II & 88 & 26 & \\
Surgery, n & III & 26 & 9 & \\
& IV & 149 & 18 & \\
& Yes & 119 & 87 & $<0.001 \mathrm{~b}^{*}$ \\
\hline
\end{tabular}

UB, Urinary Bladder; DLBCL, Diffuse large B cell lymphoma; * $p<0.05$

(a)Analysis of variance (ANOVA).

(b)Chi-square test.

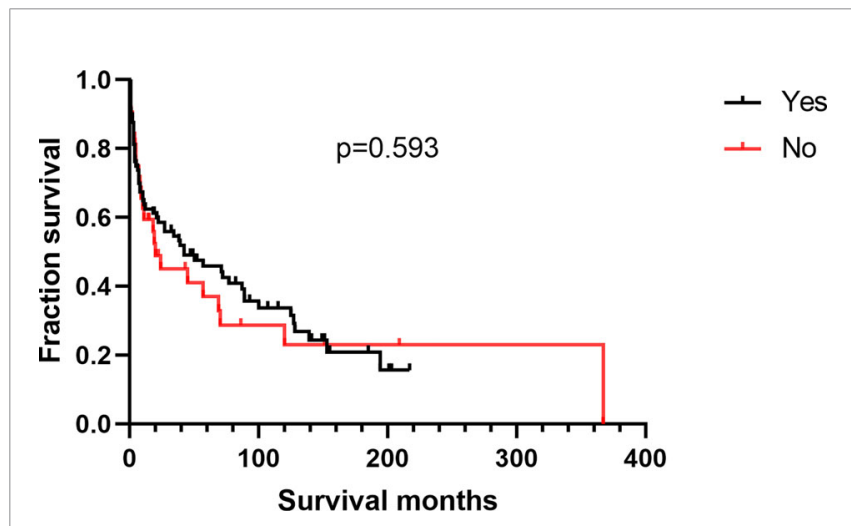

FIGURE 5 | Overall survival analysis based on surgical resection for urinary bladder DLBCL.

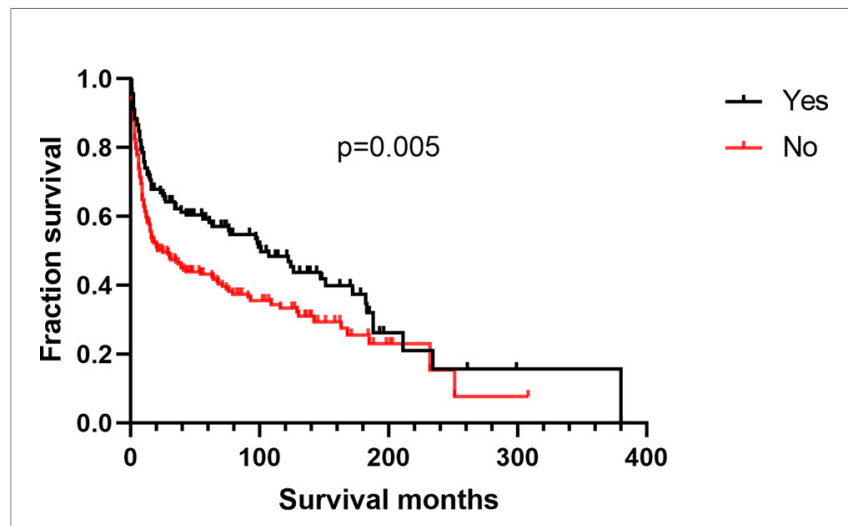

FIGURE 6 | Overall survival analysis based on surgical resection for kidney DLBCL.

Additionally, we found that the female gender was also an independent prognostic factor, which is consistent with the theory that female patients with B-cell lymphomas may have a better response to chemotherapy combined with rituximab $(14,15)$.

Furthermore, we found that surgical resection may be beneficial for patients with UT-DLBCL, especially for those with kidney DLBCL. However, there was no beneficial effect of surgery on patients with UB-DLBCL. There are no surgical recommendations for UB lymphomas, mainly because of the morbidity and mortality associated with surgery (16). Regarding radiotherapy, our results suggested that radiation treatment had no benefit on UT-DLBCL patients. Notably, this result may be biased because there are inherent limitations of SEER radiotherapy data. Radiation therapy is an important treatment for nodal DLBCL, and the International Lymphoma Radiotherapy Group includes in its guidelines that "pelvic lymphomas involve the bladder or gynaecological organs" and recommends the use of radiation therapy. However, no specific indications have been outlined for kidney lymphomas (17). Thus, the effect of radiation therapy needs to be further explored in more targeted research.

Chemotherapy, as the most used treatment in this study population, can significantly improve prognosis. Chemotherapy is still the mainstay of nodal DLBCL treatment (18). However, a previous study showed that the primary site of extranodal NHL determines the effectiveness of chemotherapy; they found no significant improvement in patients with genitourinary DLBCL who had undergone chemotherapy with rituximab (19). The dissension may be attributed to the confounding of the surgery, as there are no data on the use of combination with other therapy. Thus, further studies are urgently needed to clarify the survival benefits of chemotherapy in patients with UT-DLBCL.

There were several differences between the UB and kidney DLBCL groups. Similar to the previous results, patients with UB-DLBCL were predominantly female, whereas those with kidney DLBCL were predominantly male (13). More than half of the cases of UB-DLBCL (56.56\%) were diagnosed at Stage I, compared with only $25.71 \%$ of the cases of kidney DLBCL. The chief complaints in patients with UB-DLBCL were mainly haematuria, and this may have facilitated early diagnosis (20). More patients with UB-DLBCL than those with DLBCL underwent surgical resection. Patients with UB-DLBCL have more access to excisional biopsy than those with kidney tumours.

This study has several limitations, including a lack of data on the treatments, especially chemotherapy and combined therapy. Thus, future research involving a larger population is needed to confirm the findings.

\section{CONCLUSION}

This is the first study to demonstrate the effect of demographic and clinical features of patients with UT-DLBCL on outcome prediction. Advanced age, male gender, and Stage IV were all important poor prognostic factors for primary UT-DLBCL. Surgical resection may be beneficial for UT-DLBCL. Chemotherapy was the mainstay of treatment of patients with UT-DLBCL.

\section{DATA AVAILABILITY STATEMENT}

Publicly available datasets were analyzed in this study. This data can be found here: Surveillance, Epidemiology, and End Results (SEER) database (https://seer.cancer.gov/). 


\section{AUTHOR CONTRIBUTIONS}

Z-HL, L-CY and JZ conceived and designed the study, collected and analysed the data, and wrote the manuscript. PS and KF analysed the data. Z-FP and QD reviewed and edited the manuscript. All authors contributed to the article and approved the submitted version.

\section{REFERENCES}

1. Morton LM, Turner JJ, Cerhan JR, Linet MS, Treseler PA, Clarke CA, et al. Proposed Classification of Lymphoid Neoplasms for Epidemiologic Research From the Pathology Working Group of the International Lymphoma Epidemiology Consortium (Interlymph). Blood (2007) 110:695-708. doi: 10.1182/blood-2006-11-051672

2. Zucca E, Roggero E, Bertoni F, Cavalli F. Primary Extranodal non-Hodgkin's Lymphomas. Part 1: Gastrointestinal, Cutaneous and Genitourinary Lymphomas. Ann Oncol Off J Eur Soc Med Oncol (1997) 8:727-37. doi: 10.1023/A:1008282818705

3. Freeman C, Berg JW, Cutler SJ. Occurrence and Prognosis of Extranodal Lymphomas. Cancer (1972) 29:252-60. doi: 10.1002/1097-0142(197201) 29:1<252::AID-CNCR2820290138>3.0.CO;2-\#

4. Venyo AK. Lymphoma of the Urinary Bladder. Adv Urol (2014) 2014:327917. doi: $10.1155 / 2014 / 327917$

5. Yin X, Xu A, Fan F, Huang Z, Cheng Q, Zhang L, et al. Incidence and Mortality Trends and Risk Prediction Nomogram for Extranodal Diffuse Large B-Cell Lymphoma: An Analysis of the Surveillance, Epidemiology, and End Results Database. Front Oncol (2019) 9:1198. doi: 10.3389/fonc.2019.01198

6. Sehn LH, Donaldson J, Chhanabhai M, Fitzgerald C, Gill K, Klasa R, et al. Introduction of Combined CHOP Plus Rituximab Therapy Dramatically Improved Outcome of Diffuse Large B-Cell Lymphoma in British Columbia. J Clin Oncol Off J Am Soc Clin Oncol (2005) 23:5027-33. doi: 10.1200/JCO.2005.09.137

7. Chihara D, Oki Y, Fanale MA, Westin JR, Nastoupil LJ, Neelapu S, et al. Stage I non-Hodgkin Lymphoma: Difference in Survival Outcome by Primary Extranodal Site of Involvement. Br J Haematol (2019) 185:334-8. doi: 10.1111/bjh.15444

8. Castillo JJ, Winer ES, Olszewski AJ. Sites of Extranodal Involvement are Prognostic in Patients With Diffuse Large B-Cell Lymphoma in the Rituximab Era: An Analysis of the Surveillance, Epidemiology and End Results Database. Am J Hematol (2014) 89:310-4. doi: 10.1002/ajh.23638

9. Armitage P, Doll R. The Age Distribution of Cancer and a Multi-Stage Theory of Carcinogenesis. Br J Cancer (2004) 91:1983-9. doi: 10.1038/sj.bjc.6602297

10. Fougère B, Boulanger E, Nourhashémi F, Guyonnet S, Cesari M. Chronic Inflammation: Accelerator of Biological Aging. J Gerontol Ser A Biol Sci Med Sci (2017) 72:1218-25. doi: 10.1093/gerona/glw240

11. Weyand CM, Fulbright JW, Goronzy JJ. Immunosenescence, Autoimmunity, and Rheumatoid Arthritis. Exp Gerontol (2003) 38:833-41. doi: 10.1016/ S0531-5565(03)00090-1

\section{FUNDING}

The work is supported by the 1.3.5 project for disciplines of excellence, West China Hospital, Sichuan University (ZY2016104) and the National Natural Science Fund of China (81771569).

12. Rosenwald A, Wright G, Chan WC, Connors JM, Campo E, Fisher RI, et al. The Use of Molecular Profiling to Predict Survival After Chemotherapy for Diffuse large-B-Cell Lymphoma. N Engl J Med (2002) 346:1937-47. doi: 10.1056/NEJMoa012914

13. Lontos K, Tsagianni A, Msaouel P, Appleman LJ, Nasioudis D. Primary Urinary Tract Lymphoma: Rare But Aggressive. Anticancer Res (2017) 37:6989-95. doi: 10.21873/anticanres.12167

14. Ngo L, Hee SW, Lim LC, Tao M, Quek R, Yap SP, et al. Prognostic Factors in Patients With Diffuse Large B Cell Lymphoma: Before and After the Introduction of Rituximab. Leukemia Lymphoma (2008) 49:462-9. doi: 10.1080/10428190701809156

15. Riihijärvi S, Taskinen M, Jerkeman M, Leppä S. Male Gender is an Adverse Prognostic Factor in B-Cell Lymphoma Patients Treated With Immunochemotherapy. Eur J Haematol (2011) 86:124-8. doi: 10.1111/j.1600-0609.2010.01541.x

16. Xu H, Chen Z, Shen B, Wei Z. Primary Bladder Mucosa-Associated Lymphoid Tissue Lymphoma: A Case Report and Literature Review. Medicine (2020) 99: e20825. doi: 10.1097/MD.0000000000020825

17. Raderer M, Kiesewetter B, Ferreri AJ. Clinicopathologic Characteristics and Treatment of Marginal Zone Lymphoma of Mucosa-Associated Lymphoid Tissue (MALT Lymphoma). CA: Cancer J Clin (2016) 66:153-71. doi: 10.3322/caac. 21330

18. Armitage JO. How I Treat Patients With Diffuse Large B-Cell Lymphoma. Blood (2007) 110:29-36. doi: 10.1182/blood-2007-01-041871

19. Olszewski AJ, Winer ES, Castillo JJ. Improved Survival With Rituximab-Based Chemoimmunotherapy in Older Patients With Extranodal Diffuse Large B-Cell Lymphoma. Leukemia Res (2014) 38:866-73. doi: 10.1016/j.leukres.2014.04.009

20. Simpson WG, Lopez A, Babbar P, Payne LF. Primary Bladder Lymphoma, Diffuse Large B-Cell Type: Case Report and Literature Review of 26 Cases. Urol Ann (2015) 7:268-72. doi: 10.4103/0974-7796.152947

Conflict of Interest: The authors declare that the research was conducted in the absence of any commercial or financial relationships that could be construed as a potential conflict of interest.

Copyright (c) 2021 Liu, Yang, Song, Fang, Zhou, Peng and Dong. This is an openaccess article distributed under the terms of the Creative Commons Attribution License (CC BY). The use, distribution or reproduction in other forums is permitted, provided the original author(s) and the copyright owner(s) are credited and that the original publication in this journal is cited, in accordance with accepted academic practice. No use, distribution or reproduction is permitted which does not comply with these terms. 